MATEC Web of Conferences 13,02029 (2014)

DOI: $10.1051 /$ matecconf/ 20141302029

(C) Owned by the authors, published by EDP Sciences, 2014

\title{
Numerical Study of Water Control with Downhole Oil-Water Separation Technology
}

\author{
Khor Yin Yin ${ }^{1, a}$, Hussain H. Al-Kayiem², William Pao ${ }^{2}$ \\ 1,2,3 Universiti Teknologi PETRONAS, Mechanical Engineering Department, block 17, Bandar Seri \\ Iskandar, 31750 Tronoh, Malaysia
}

\begin{abstract}
The maturing oil fields with increasing water production can pose a challenging produced water handling and disposal issues. This paper presents a numerical study of a motorless hydrocyclone to enhance understanding of the downhole oil-water separation. The turbulence of fluid flow is obtained using K- $\varepsilon$ Realizable Turbulence model for complex swirl dominated flow, while the interface between hydrocarbon and water is described using the Discrete Phase model. In this approach, factors which contribute to the hydrocyclone separation instability were discussed. Discussion is then extended to the relationship of residence time with pressure difference between overflow and underflow. These pressure differences are able to relate to pressure condition for high water cut well which require downhole separation.
\end{abstract}

\section{Introduction}

An increasing water production in oil and gas wells due to water influx has encouraged the industry to investigate solution to minimize the impact of concurrent production of unwanted water in the wells. In the recent decade, there is growing recognition for the need of a controlled water production to meet the immense challenges of increasing water handling cost and to extend the life of the well especially at offshore operation. Virtually, water production in mature field is unavoidable; oil is commonly accompanied by an underlying aquifer, as production rate is increased, oil water contact (OWC) also increases until water breakthrough into the wellbore. The unavoidable premature water breakthrough or water coning could happen due to high pressure drawdown around the wellbore and high mobility of water than oil, where water viscosity much lesser than the oil, water relative permeability much greater than oil. The consequences of increased water production translates to high operating expenses and produced water disposal costs, increased in environmental risk associated with large volumes of produced water, additional requirement for produced water lifting and significantly reduced surface processing facilities ullage for oil. Water production can also limit the well production life via fines migrations and high water production inhibits oil production to production tubing, hence causing low recovery at oil layer.

These lead to a solution to the problem of unwanted water production; the development of Downhole Oil-Water Separation (DOWS) technology. DOWS is a hydrocyclone-based system for downhole separation of produced oil and water and subsequent disposal of the produced water by reinjection within the same wellbore using electric submersible pump. It has been successfully applied in a number of onshore wells, for example Alberta Canada, France, North Sea and China [1]. However, based on lessons learned from onshore and offshore installations, the used of DOWS in

a Corresponding author : khoryin_yin@petronas.com.my

This is an Open Access article distributed under the terms of the Creative Commons Attribution License 2.0, which permits unrestricted use, distribution, and reproduction in any medium, provided the original work is properly cited. 
offshore is limited due to its complex apparatus set up, space limitation for power generation and difficulty to re-enter well for troubleshooting. Nevertheless, this paper will focus on the exploitation of downhole separation without the use of electric submersible pump. Theoretically such concept requires relative positioning of the zones of a high pressure production layers on top and a low pressure water zone below to complete hydrocyclone separation as implemented in oil field Malaysia.

In the past, many efforts have been made to study the flow behavior in hydrocyclone. Experimental investigation and related empirical models were set up to cover ranges of operating condition and hydrocyclone geometry, for example Liquid-Liquid cylindrical cyclone for inlet velocity below $0.8 \mathrm{~m} / \mathrm{s}$ [2]. However, these experimental approaches are found to be expensive and empirical models are restricted to the parameters which were used in the experiments.

In this study, computational fluid dynamics (CFD) is adopted to obtain quantitative description of separation performance at different flow and operational conditions. With the development of CFD, it is now possible to computationally assess the performance of hydrocyclone and evaluate design optimization prior to implementation of costly experimental investigation. The hydrocyclone was modeled and simulated using ANSYS-Fluent 14 commercial software to study the effect of residence time versus pressure difference between overflow and underflow.

\section{Fundamentals of design}

The principle of hydrocyclone separation is practically straight forward. A schematic diagram of hydrocyclone is shown in Figure 1. The pressurized oil-water mixture is fed tangentially (horizontal inlet) into the vertical cylindrical part, forcing a strong swirling motion within the cyclone. Note that small inlet will encourage droplet break-up while big inlet will lead to insufficient swirl intensity [2]. The conical shape of the hydrocyclone accelerates the mixture flow into a helical pattern, setting up a free vortex and creating large centrifugal forces. The cylindrical part gives some residence time for the particles to experience centrifugal force, so that denser material can migrate toward the wall. The cone further constricts the downward flow, creating a central spiralling upward flow. The narrower the cone angle, the smaller percentage of secondary phase escapes from underflow. However, the narrow cone angle will lead to higher pressure drop. The oil-rich stream exits through the top overflow outlet and a water-rich stream leaves the system through the bottom underflow outlet.

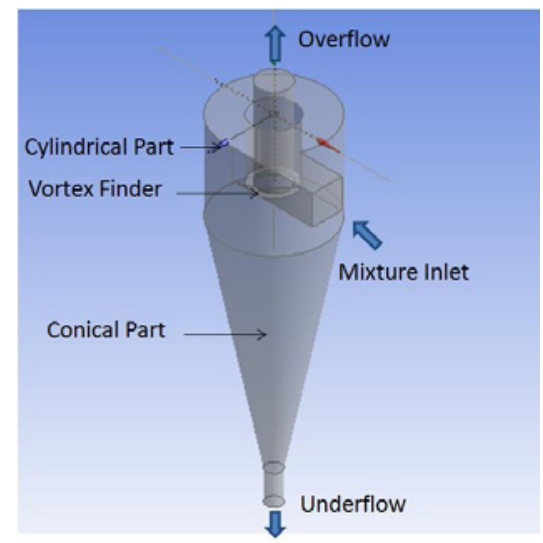

Figure 1. Basic design of a hydrocyclone

In the aquifer supported oil reservoir, density, surface tension and water phase salinity are key factor for effective separation. Separation efficiency increase with higher density due to oil droplet terminal velocity is higher and separation consequently more efficient. To achieve maximum density difference in the system, the most favourable condition is at higher temperature and at bubble point pressure due oil is at its minimum density. Furthermore, if formation water is high salt content, it will also improve separation due to higher surface tension and thereby coalescence between droplets is 
enhanced together with higher density of salt water [3]. This paper will analyses the feasibility of downhole technologies that used to restrict water from entering the wellbore then to surface. A motorless concept will be focused in this research work utilizing the pressure difference between sand layers to produce sufficient energy for oil water separation.

The hydro cyclone shown in Figure 2 which was experimentally studied in 1980 has been a great reference for liquid-liquid hydrocyclone model. This design has inlet chamber and the reducing section to achieve higher tangential acceleration of the fluid, reducing the pressure drop and the shear stress to an acceptable level. Most of the separation is achieved at the tapered section, where low angle segment keeps the swirl intensity with high residence time. Finally a long tail pipe cylindrical section in which the smallest droplets migrate to the reversed flow core at the axis and are being separated into the overflow exit [4].

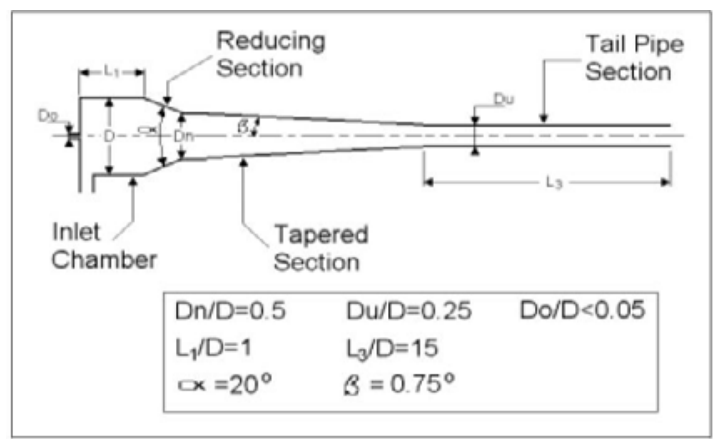

Figure 2. Hydrocyclone Geometry, $\mathrm{D}=60 \mathrm{~mm}$

Dimension of this standard hydrocyclone with dual tangential inlet as shown in Figure 2 is used due to this design has been widely reported by several researchers for model validation.

\section{Numerical Model}

Numerical analysis is presented in detail to manipulate the hydrocyclone to achieve the desire performance. Computer simulations were carried out with AYSYS-Fluent version 14.5. The stated model was solved using three-dimensional unstructured hexahedral. In order to capture the reversed flow at the hydrocyclone, block structured mesh is generated using ICM. This model is consisting of 165696 cells as shown in Figure 3. Boundary conditions are based on analytical analysis and tests carried out in the field. Assuming isothermal and steady state condition, the inlet boundary conditions are an inflow with a constant velocity of $2 \mathrm{~m} / \mathrm{s}$ with pressure at inlet is $620.532 \mathrm{kPa}(90 \mathrm{psi})$ and outlet underflow is $434.372 \mathrm{kPa}(63 \mathrm{psi})$. The primary phase is water with a density of $1000.0 \mathrm{~kg} / \mathrm{m}^{3}$ and a viscosity value of $0.00097 \mathrm{~kg} / \mathrm{m} \bullet \mathrm{s}$, while the inert liquid spherical particles, which is oil in this case has density and viscosity of $840 \mathrm{~kg} / \mathrm{m}^{3}$ and $0.00136 \mathrm{~kg} / \mathrm{m} \bullet \mathrm{s}$ respectively. The oil droplet sizes are ranging from $2.3 \mu \mathrm{m}$ to $200 \mu \mathrm{m}$, hence the mean drop size is $51 \mu \mathrm{m}$. In general, larger droplets move faster and increase cyclone efficiency [3].

Discrete Phase Modeling (DPM) technique was adopted to achieve the particle separation behavior using Lagrangian approaches. This method simulated the paths of individual particles through the fluid, which is calculated from a force balance on a particle. To characterize the fluid dynamics of the hydrocyclone, the key component is the turbulence model. Simulations with the k$\varepsilon$ Standard Turbulence model, Renormalization Group (RNG), Reynolds Stress Model (RSM) or superior models as LES are commonly studied by researcher. The k- $\varepsilon$ Realizable Turbulence model was shown to have the capability of reproducing complex swirling flows, reaching transitory oscillatory solutions [6].

As a result, k- $\varepsilon$ Realizable Turbulence model is chosen as the closure model. For Pressure-Velocity coupling, SIMPLE algorithm is used while First Order Upwind scheme is applied for the momentum. 
As for pressure interpolation scheme, PRESTO! (PREssure STaggering Option) scheme is adopted. These selections were the recommended scheme in order to improve the estimation for highly swirling flow simulations. The simulations are assumed to be converged when the residual difference was smaller than 10-3 for all variables.

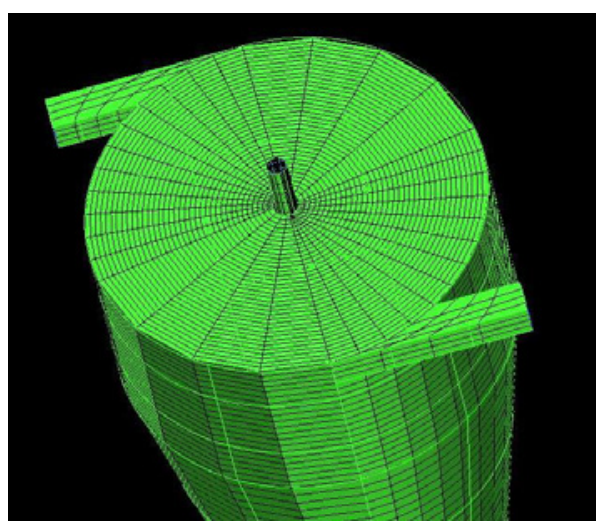

Figure 3. Image of grid used in CFD simulation

\section{Results and Discussion}

It is necessary to validate the model before its application for numerical experiment. In the simulation for base case, the inlet velocity is $2 \mathrm{~m} / \mathrm{s}$, oil density is $840 \mathrm{~kg} / \mathrm{m} 3$ and $90 \%$ water cut, this means the volume fraction of second phase (oil) is 10 percent. Figure 4 describes the flow field in a hydrocyclone numerically. As illustrated in the velocity profile, the mixtures are tangentially injected into the hydrocyclone through the horizontal inlet and cause a swirling motion inside the conical part. Lighter fluid is expected to be separated and flow through overflow outlet while heavier fluid discharged through underflow outlet due to the effect of centrifugal force. Figure 4 also shows that the vortex is helical twisted cylinder and not completely an axially geometrical symmetry.
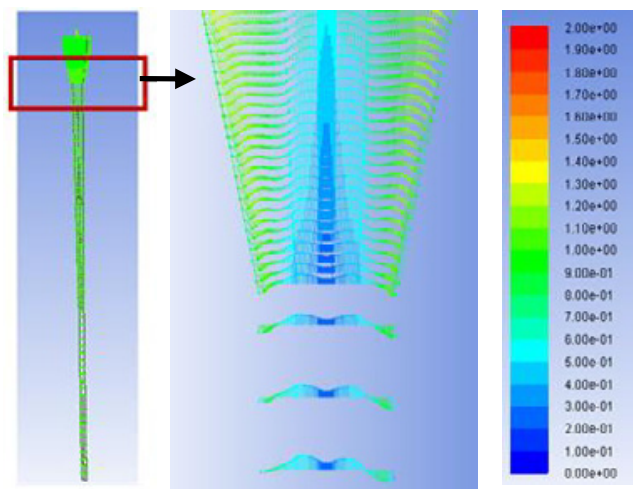

Figure 4. Tangential Velocity $[\mathrm{m} / \mathrm{s}]$ distribution profile

Effect of pressure difference can be evaluated as illustrated in the plot in Figure 5. Oil droplet separation is a result of the force imposed on the oil droplet and the residence time in the hydrocyclone. Supposedly lower flow rate has higher residence time and higher flow rat has lower residence time. The simulation is able to match the impact of delta pressure between overflow and underflow. This figure shows that there is no direct relationship for residence time and pressure delta. All of these 3 models has shown 100\% separation efficiency, where underflow outlet is getting $100 \%$ water. Tracking of the oil droplets is shown in Figure 6. 


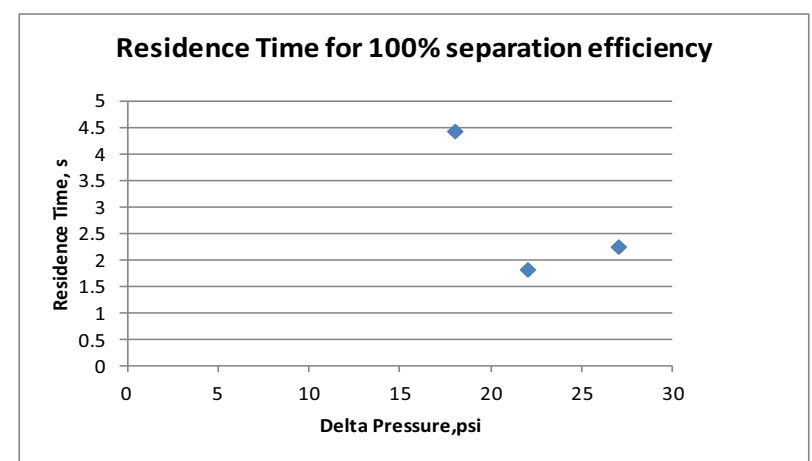

Figure 5. Residence time [s] versus Pressure difference [psi]

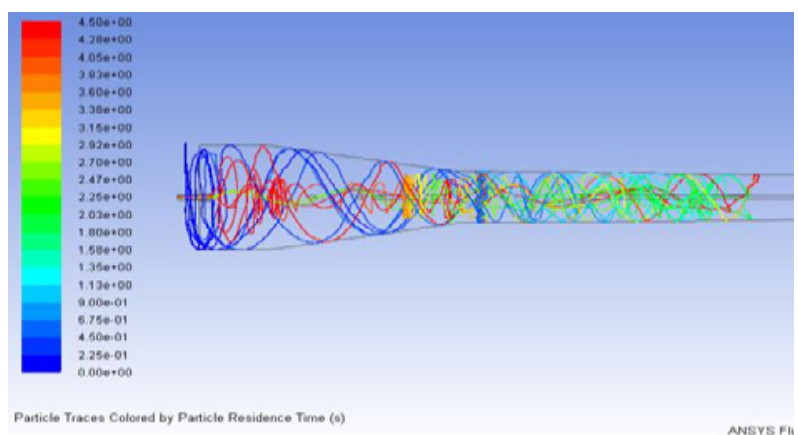

Figure 6. Pathlines showing the single oil particle spiral flow in a cyclone colored by residence time Conclusion and Way Forward

\section{Conclusions}

A full-scale of oil-water hydrocyclone is modeled and simulated by Computational Fluid Dynamics technique in 3-dimenssional, steady assumption. The model can become an essential tool in order to determine the separation efficiency for different ranges of parameter. The simulation at the existing parameter has shown 100\% separation efficiency. This CFD base model can also determine the separation performance when many inlets or different configurations are adopted in the hydrocyclone.

\section{Acknowledgment}

The authors acknowledge Universiti Teknologi PETRONAS for sponsoring the research under the YUTP-FRG 015-3AA-A34 research grants. Also, the support from PETRONAS-Sarawak Resource Management is highly appreciated.

\section{References}

1. Y. K. Bangash, SPE Wood Group ESP, Inc. SPE 81123, (2003)

2. C. Oropeza-Vazquez, E. Afanador, L. Gomez, S. Wang, R. Mohan, O. Shoham, G. Kouba,. University of Tulsa, Tulsa.(2004)

3. K.B. Yasser, R.B Michael, E.C. Stephen, Wood Group ESP Inc., Oklahoma City, US. US 6547003 B1. (2000)

4. C.H. Gomez, University of Tulsa, Tulsa. (2001)

5. M. Carmona, C. Cortes and A. Ramirez, Spain. (2010) 